\title{
Síntesis de un pigmento rojo a partir de nanopartículas de oro
}

\author{
S. MESTRE, M. D. PALACIOS, P. AGUT
}

Instituto de Tecnología Cerámica (ITC). Asociación de Investigación de las Industrias Cerámicas (AICE). Universitat Jaume I. Castellón. España.

Este trabajo ha sido presentado como comunicación oral, tras su evaluación por el Comité Científico, en el XII Foro Global del Recubrimiento Cerámico. QUALICER (13 y 14 febrero 2012. Castellón. España).

\begin{abstract}
Se ha desarrollado un método simple para sintetizar pigmentos rojos para vidriados cerámicos, basados en nanopartículas de oro protegidas por una cápsula de óxido refractario. Las nanopartíclas de oro con el tamaño adecuado se han obtenido por reacción en medio acuoso entre una disolución de $\mathrm{Au}(\mathrm{III})$ y otra de bromuro amónico, manteniendo reducida la concentración de $\mathrm{Br}^{-}$durante el proceso.

El encapsulado de las nanopartículas áureas se ha conseguido adicionando el óxido protector en forma de coloide y coagulándolo a continuación alcalinizando el medio. Posteriormente se incorpora dietilentriamina para favorecer la interacción entre las nanopartículas áureas y las oxídicas, y posteriormente carboximetilcelulosa para elevar la viscosidad del medio, y evitar la segregación y posterior aglomeración de las nanopartículas de oro durante el secado. El residuo seco se puede utilizar directamente como pigmento en vidriados, sin necesidad de tratamientos térmicos posteriores.

Se han ensayado tres óxidos protectores, $\mathrm{SiO}_{2}, \mathrm{Al}_{2} \mathrm{O}_{3}$ y $\mathrm{SnO}_{2}$, obteniéndose en los tres casos pigmentos con un elevado poder colorante, y que generan rojos con diferentes tonalidades en el vidriado ensayado.
\end{abstract}

Palabras clave: sintesis, pigmento, nanopartículas, encapsulado.

\section{Synthesis of a red pigment from gold nanoparticles}

A simple method of synthesising red pigments for ceramic glazes, based on gold nanoparticles protected by a refractory oxide capsule, was developed. Gold nanoparticles of an appropriate size were obtained by reaction in an aqueous medium between an $\mathrm{Au}(\mathrm{III})$ solution and an ammonium bromide solution, keeping the Br concentration low during the process.

The gold nanoparticles were encapsulated by adding the protective oxide in colloid form and subsequently coagulating it, alkalinising the medium. Diethylentriamine was then added to encourage the interaction between the gold nanoparticles and the oxide nanoparticles. This was followed by adding carboxymethylcellulose to raise medium viscosity, and to avoid segregation and subsequent agglomeration of the gold nanoparticles during drying. The dry residue was directly usable as a glaze pigment without requiring further thermal treatments.

Three protective oxides, namely $\mathrm{SiO}_{2}, \mathrm{Al}_{2} \mathrm{O}_{3^{\prime}}$ and $\mathrm{SnO}_{2}$, were tested. In the three cases, pigments with a high colouring strength were obtained, which gave rise to reds of different shades in the resulting test glaze.

Keywords: synthesis, pigment, nanoparticles, encapsulation.

\section{INTRODUCCIÓN}

Uno de los colores más difíciles de conseguir en cerámica es el rojo, sobre todo en vidriados de alta temperatura. En la actualidad el sulfoseleniuro de cadmio encapsulado en circón es una solución aceptable, tanto desde el punto de vista de rendimiento de color como en estabilidad. Sin embargo, la elevada toxicidad del $\mathrm{Cd}(\mathrm{S}, \mathrm{Se})$ supone un riesgo desde el punto de vista medioambiental. Por ello es necesario disponer de pigmentos rojos alternativos, sobre todo en previsión de las restricciones que impondrá la normativa $\mathrm{REACH}$, al menos en Europa.

Las decoraciones basadas en oro coloidal se utilizan en cerámica y vidrio desde hace siglos (conocidas como Púrpura de Cassius) (1), pero su obtención es complicada, y sobre todo no se pueden utilizar como pigmentos en el sentido clásico del término, debido a su naturaleza líquida. La base física del Púrpura de Cassius es la presencia de nanopartículas de oro (AuNPs) que presentan el fenómeno denominado "Resonancia de Superficie Plasmónica" (RSP), el cual provoca absorciones muy intensas de determinadas frecuencias del espectro electromagnético (máximo en torno a $530 \mathrm{~nm}$ ) (2). Dicho fenómeno genera coloraciones rojizas muy intensas cuando las AuNPs se hallan dispersas en el seno de un vidrio, de modo que podrían ser consideradas como cromóforos inorgánicos (3). 
Se han propuesto numerosas alternativas para obtener pigmentos sólidos a partir del Púrpura de Cassius. El proceso artesanal desarrollado inicialmente constaba de numerosas etapas (precipitación del púrpura en forma de gel, mezclado del gel con vidrio molturado, calcinación del sólido y molienda final), y se caracterizaba por la escasa reproducibilidad en el poder colorante del pigmento y su falta de estabilidad a temperaturas elevadas. Por ello, a lo largo del siglo XX se han propuesto numerosas mejoras. Las primeras investigaciones se orientaron a facilitar la etapa de precipitación, y en este sentido se ha propuesto coprecipitar el coloide de oro con oxihidratos de $\mathrm{Al}$ o Sn (4), así como realizar la precipitación en presencia de materiales inertes tipo caolín o feldespato (5). Una segunda vía de investigación fue la deposición directa el coloide de oro sobre el sólido que constituiría la cápsula protectora, habiéndose propuesto como material protector fritas (6), u oxhidratos de elementos cuyos óxidos son estables en vidrios de silicato (7).

Además de las mejoras con respecto al método tradicional, se han propuesto enfoques completamente diferentes. El método más sofisticado puede ser el propuesto por Brandes et al. (8) para obtener un pigmento púrpura en forma de partículas esféricas mediante pirólisis conjunta de disoluciones que contienen el precursor del oro y el precursor de la cápsula protectora.

La presente investigación se originó al considerar que no se disponía de proceso vía húmeda fácilmente transferible a la escala industrial, que permitiese sintetizar pigmentos en el intervalo de rojos y púrpuras, los cuales presentasen un elevado poder colorante en diferentes vidriados cerámicos, incluyendo aquellos que necesitan elevadas temperaturas de cocción (gres blanco o gres porcelánico). El punto de partida fue la relativa facilidad con la que puede obtenerse una suspensión de AuNPs en medio acuoso por reducción de una sal soluble de oro $(9,10)$. El problema radicaba en encapsular las AuNPs con un óxido refractario que las protegiese de la disolución en los vidriados. Para solventarlo se planteó utilizar coloides comerciales de óxidos refractarios, ya que su tamaño de partícula nanométrico facilitaría la formación de la cápsula.

Las superficies del oro poseen una escasa afinidad por las superficies de óxido, por ello se estudió la incorporación de aditivos que pudiesen realizar la función de puente entre las AuNPs y las nanopartículas de óxido, lo cual facilitaría el encapsulado. Idealmente se requiere que un extremo de la molécula presente afinidad por el oro, y otro por los óxidos. Los tioles se unen fuertemente a la superficie de las AuNPs, y por tanto son la opción recomendada en la bibliografía para controlar el crecimiento de las nanopartículas (11). Por otra parte, las aminas también se pueden unir a la superficie de las AuNPs (12), así como a las de los óxidos. Para realizar los ensayos se escogió un representante de cada grupo. En el caso de los tioles el 6-mercaptohexanol, con el objetivo que el grupo $\mathrm{OH}$ presente en el otro extremo de la cadena facilitase la interacción con las partículas coloidales oxídicas. En el caso de las aminas se escogió la dietilentriamina, que al disponer de varios grupos amino, una misma molécula puede unirse a diferentes partículas.

\section{PROCEDIMIENTO EXPERIMENTAL}

La materia prima empleada para sintetizar las AuNPs fue el ácido tetracloroaurico trihidratado (Heraeus) y el bromuro amónico (Fluka). Como aditivos en determinados experimentos se utilizaron 6-mercaptohexanol (en adelante $6 \mathrm{MH}$, Fluka), dietilentriamina (en adelante DTA, Fluka) y una disolución de carboximetilcelulosa al $1 \%$ en peso (en adelante CMC, Hercules). Durante el desarrollo de la investigación el precursor utilizado para generar la cápsula protectora de sílice fue un coloide industrial de $\mathrm{SiO}_{2}$ en agua ( $30 \%$ en peso). Adicionalmente, para evaluar si el método de encapsulado era

TABLA I: MATERIAS PRIMAS UTILIZADAS EN LA SÍNTESIS DE LOS PIGMENTOS.

\begin{tabular}{|c|c|c|c|c|c|c|c|c|c|}
\hline Ref. & $\begin{array}{c}\mathrm{HAuCl}_{4} \\
(\mathrm{mmol})\end{array}$ & $\begin{array}{l}\mathrm{NH}_{4} \mathrm{Br} \\
(\mathrm{mmol})\end{array}$ & $\begin{array}{l}\mathrm{SiO}_{2} \\
(\mathrm{~mL})\end{array}$ & $\begin{array}{c}\mathrm{Al}_{2} \mathrm{O}_{3} \\
(\mathrm{~mL})\end{array}$ & $\begin{array}{l}\mathrm{SnO}_{2} \\
(\mathrm{~mL})\end{array}$ & $\begin{array}{l}\mathrm{NH}_{3} \\
(\mathrm{~mL})\end{array}$ & $\begin{array}{c}6 \mathrm{MH} \\
(\mu \mathrm{L})\end{array}$ & $\begin{array}{l}\text { DTA } \\
(\mu \mathrm{L})\end{array}$ & $\begin{array}{l}\text { CMC } \\
(\mathrm{mL})\end{array}$ \\
\hline A1 & 0,76 & 1,43 & 5 & - & - & 3 & - & - & - \\
\hline A2 & 1,52 & 2,86 & 20 & - & - & 12 & - & - & - \\
\hline B1 & 0,76 & 1,43 & 5 & - & - & 3 & 100 & - & - \\
\hline B2 & 0,76 & 1,43 & 5 & - & - & 3 & 100 & - & - \\
\hline B3 & 0,76 & 1,43 & 5 & - & - & 3 & 100 & - & - \\
\hline C1 & 0,76 & 1,43 & 5 & - & - & 3 & - & 100 & - \\
\hline D1 & 0,76 & 1,43 & - & 10 & - & 3 & - & 100 & 32 \\
\hline D2 & 0,76 & 1,43 & - & - & 13 & 3 & - & 100 & 32 \\
\hline
\end{tabular}


extensible a otros óxidos, se utilizaron coloides comerciales de $\mathrm{Al}_{2} \mathrm{O}_{3}\left(20 \%\right.$ en peso, Alfa Aesar) y $\mathrm{SnO}_{2}$ ( $15 \%$ peso, Alfa Aesar). $\mathrm{El}$ poder colorante de los diferentes pigmentos sintetizados se evaluó en un vidriado transparente de monococción, obtenido a partir de una frita industrial tipo cristalina.

En líneas generales, la síntesis de las diferentes muestras de pigmento se realizó partiendo de $100 \mathrm{~mL}$ de agua destilada, y cantidades de ácido tetracloroaurico y bromuro amónico que mantuvieron una relación molar 0,53 aproximadamente (Tabla I). Los dos reactantes y el agua se combinaron de distintos modos para mantener diferentes concentraciones de precursores durante la reacción (Tabla II), y posteriormente se mantuvieron en contacto un tiempo determinado $\left(t_{R}\right)$, con el objetivo de obtener una suspensión estable de AuNPs con el tamaño adecuado. A la suspensión recién preparada se adicionó el coloide del óxido escogido en cada caso y finalmente amoníaco para provocar la gelificación del coloide y la formación de la cápsula protectora (se adicionaron $0,6 \mathrm{~mL}$ de $\mathrm{NH}_{3} 35 \%$ por cada $\mathrm{mL}$ de coloide incorporado). A lo largo del desarrollo se ensayaron los aditivos citados previamente, en función de cuya naturaleza y comportamiento se reajustó el procedimiento general.

La suspensión obtenida en cada caso se secó bajo lámparas de infrarrojos, y opcionalmente se sometió a una calcinación de 30 minutos a $900^{\circ} \mathrm{C}$ para afianzar la cápsula protectora sobre las AuNPs. El pigmento obtenido se disgregó en un mortero de ágata y se tamizó en una malla de $200 \mu \mathrm{m}$, pero no se sometió a molturación para evitar la rotura de la cápsula de óxido generada. En muestras seleccionadas, se investigó el tamaño y la morfología de las AuNPs presentes en las partículas de pigmento mediante microscopía electrónica de barrido (FEG-SEM Quanta 200F, FEI Co, USA).

$\mathrm{El}$ primer bloque de experimentos (A1 y A2) fue diseñado para evaluar el efecto del tiempo de reacción y la proporción $\mathrm{Au} / \mathrm{SiO}_{2}$ sobre el poder colorante del pigmento, así como la viabilidad de obtener el pigmento sin incorporar aditivos orgánicos, ya que en el caso de ser factible, sería un proceso mucho más simple de escalar.

El segundo bloque de experimentos (B1, B2 y B3), se diseñó para investigar los efectos de dos parámetros. En primer lugar la concentración de los precursores durante la formación de las AuNPs. En segundo lugar la incorporación de $6 \mathrm{MH}$ como agente para controlar el tamaño de dichas nanopartículas, y favorecer su unión con las nanopartículas de sílice. Los experimentos se realizaron utilizando las mismas cantidades de reactantes que en el A1, pero adicionando el $6 \mathrm{MH}$ en cantidad equimolar con el oro. En el B1 se mantuvo reducida la concentración de $\mathrm{NH}_{4} \mathrm{Br}$ con respecto al $\mathrm{HAuCl}_{4}$ durante la reacción, en el B2 se invirtieron las concentraciones y en el B3 se mantuvieron reducidas las concentraciones de ambos precursores.

TABLA II: CONDICIONES DE OPERACIÓN EMPLEADAS DURANTE LA SÍNTESIS DE LOS PIGMENTOS.

\begin{tabular}{|c|c|c|c|}
\hline Ref. & CARACTERÍSTICAS ESPECÍFICAS DE LA SÍNTESIS & $\begin{array}{c}\begin{array}{c}t_{\mathrm{R}} \\
(\mathrm{min})\end{array} \\
\end{array}$ & CALCINACIÓN \\
\hline A1 & Adición del $\mathrm{NH}_{4} \mathrm{Br}$ sólido a la disolución de $\mathrm{HAuCl}_{4}$ & 5 & $\mathrm{Si}$ \\
\hline A2 & Idéntico al A1 & 60 & $\mathrm{Si}$ \\
\hline B1 & $\begin{array}{c}\text { Adición gota a gota de } 50 \mathrm{~mL} \text { de una disolución conteniendo el } \mathrm{NH}_{4} \mathrm{Br} \text { y el } 6 \mathrm{MH} \text { sobre } 50 \mathrm{~mL} \text { de una } \\
\text { disolución conteniendo el } \mathrm{HAuCl}_{4} \text {. }\end{array}$ & 5 & $\mathrm{Si}$ \\
\hline B2 & $\begin{array}{l}\text { Adición gota a gota de } 50 \mathrm{~mL} \text { de una disolución conteniendo el } \mathrm{HAuCl}_{4} \text { sobre } 50 \mathrm{~mL} \text { de una } \\
\text { disolución conteniendo el } \mathrm{NH}_{4} \mathrm{Br} \text { y el } 6 \mathrm{MH}\end{array}$ & 5 & $\mathrm{Si}$ \\
\hline B3 & $\begin{array}{l}\text { Adición gota a gota simultáneamente de } 10 \mathrm{~mL} \text { de dos disoluciones, una conteniendo el } \mathrm{HAuCl}_{4} \text { y } \\
\qquad \text { otra el } \mathrm{NH}_{4} \mathrm{Br} \text { y el } 6 \mathrm{MH} \text {, sobre } 80 \mathrm{~mL} \text { de } \mathrm{H}_{2} \mathrm{O}\end{array}$ & 5 & $\mathrm{Si}$ \\
\hline $\mathrm{C} 1$ & $\begin{array}{l}\text { Adición gota a gota de } 50 \mathrm{~mL} \text { de una disolución conteniendo el } \mathrm{NH}_{4} \mathrm{Br} \text { sobre } 50 \mathrm{~mL} \text { de una disolución } \\
\text { conteniendo el } \mathrm{HAuCl}_{4} . \\
\text { Adición de DTA después del amoníaco }\end{array}$ & 5 & $\mathrm{Si}$ \\
\hline $\mathrm{C} 2$ & $\begin{array}{l}\text { Adición gota a gota de } 50 \mathrm{~mL} \text { de una disolución conteniendo el } \mathrm{NH}_{4} \mathrm{Br} \text { sobre } 50 \mathrm{~mL} \text { de una disolución } \\
\text { conteniendo el } \mathrm{HAuCl}_{4} \text {. } \\
\text { Adición del DTA y de la CMC después del amoníaco }\end{array}$ & 5 & No \\
\hline D1 & Idéntico al C2 & 5 & No \\
\hline D2 & Idéntico al C2 & 5 & No \\
\hline
\end{tabular}


El tercer bloque de experimentos (C1 y C2), se concibió principalmente para analizar los efectos de la incorporación de DTA sobre la formación de la cápsula protectora. Paralelamente se analizaron los efectos de la viscosidad del medio durante el secado y de la calcinación final. En ambos experimentos la síntesis se realizó manteniendo reducida la concentración de $\mathrm{NH}_{4} \mathrm{Br}$ durante la reacción e incorporando un $10 \%$ molar de DTA respecto al oro. Sin embargo, el DTA se introdujo después de incorporar el amoníaco para favorecer la formación de puentes entre las AuNPs y la sílice, y evitar en la medida de lo posible que los puentes se formasen entre las propias AuNPs. En el experimento C2 también se incorporó la disolución de CMC al medio para incrementar su viscosidad y dificultar la segregación de las partículas durante el secado, y además no se realizó la calcinación, para evaluar si durante la misma se producía el crecimiento de las AuNPs.

El cuarto bloque de experimentos (D1 y D2) fue diseñado para evaluar si el proceso que conducía al pigmento $\mathrm{C} 2$ era generalizable a cápsulas de otros óxidos, para lo cual se ensayaron coloides de $\mathrm{Al}_{2} \mathrm{O}_{3}$ y $\mathrm{SnO}_{2}$ como precursores de la cápsula protectora, si bien hubo que reajustar los volúmenes de coloide en función de su concentración, para mantener aproximadamente constante la relación Au/óxido (en peso).

Los pigmentos se incorporaron al vidriado transparente, aplicando las barbotinas de esmalte sobre soportes bizcochados de cocción blanca, con un patín de $600 \mu \mathrm{m}$ de paso. Una vez secas, las probetas se cocieron con un tratamiento térmico tipo pavimento de monococción, caracterizado por una temperatura máxima de $1120{ }^{\circ} \mathrm{C}$ y un tiempo de permanencia a la misma de 6 minutos. La medida de las coordenadas cromáticas CIELab de los vidriados obtenidos se realizó con un espectrofotómetro de reflectancia difusa (Color Eye 7000A, $\mathrm{X}$-Rite Inc. USA), empleando el iluminante estándar CIE $\mathrm{D}_{65} \mathrm{y}$ el observador estándar CIE $10^{\circ}$.

En esta investigación los pigmentos se incorporaron al vidriado en una proporción variable, ya que la cantidad de muestra disponible cambió notablemente entre los distintos experimentos. Además, como la proporción de oro en los pigmentos presentó un intervalo de variación relativamente amplio, se ha tomado como referencia el porcentaje teórico de oro que se introdujo en el esmalte seco en cada caso (que varió entre un $0,05 \%$ y un $0,2 \%$ dependiendo del experimento), ya que este elemento es el cromóforo real. Por esta misma razón, se ha definido una estimación del rendimiento del oro incorporado, para comparar el poder colorante de los pigmentos sintetizados. Se definió como factor de desarrollo del oro $(\Psi)$, el cociente entre el incremento de la componente roja generada en el vidriado y el porcentaje en peso teórico de oro que contenía dicho vidriado.

$$
\Psi=\frac{a^{*}-a_{\text {vidriado base }}^{*}}{\% A u}
$$

Obviamente la relación entre las coordenadas cromáticas y el porcentaje de oro no es lineal, ya que a partir de cierto porcentaje de oro se alcanza la saturación del color. Sin embargo, durante la investigación se trabajó con concentraciones muy bajas de cromóforo, de modo que se consideró que se estaría dentro del intervalo donde la relación es aproximadamente lineal, y por tanto $\psi$ sería significativo para discriminar resultados de diferentes experimentos.

\section{RESULTADOS Y DISCUSIÓN}

\subsection{Síntesis de los pigmentos}

Durante la síntesis de los dos primeros pigmentos (A1 y A2) se apreció que la disolución amarilla de $\mathrm{HAuCl}_{4}$ viraba rápidamente al rojo intenso tras la adición del $\mathrm{NH}_{4} \mathrm{Br}$, sin que se perdiese la transparencia. Este cambio podría deberse a la aparición de las AuNPs debido a un efecto reductor del bromuro, pero también existía la posibilidad de que

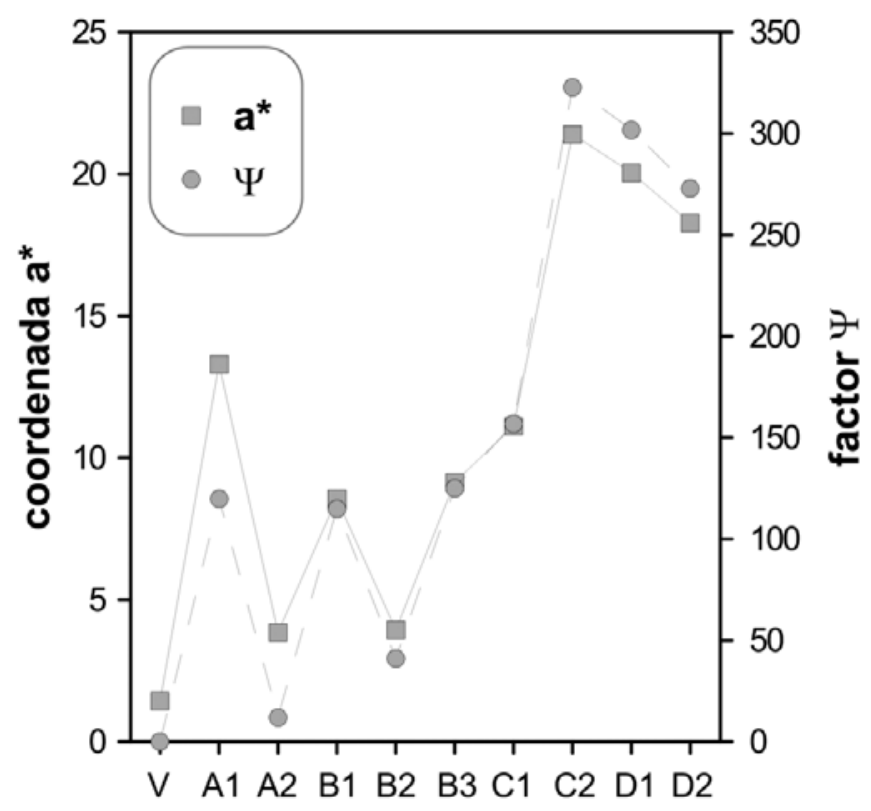

Figura 1: Coordenada $a^{*}$ y factor $\Psi$ del vidriado transparente, y de los vidriados que incorporaban los pigmentos.

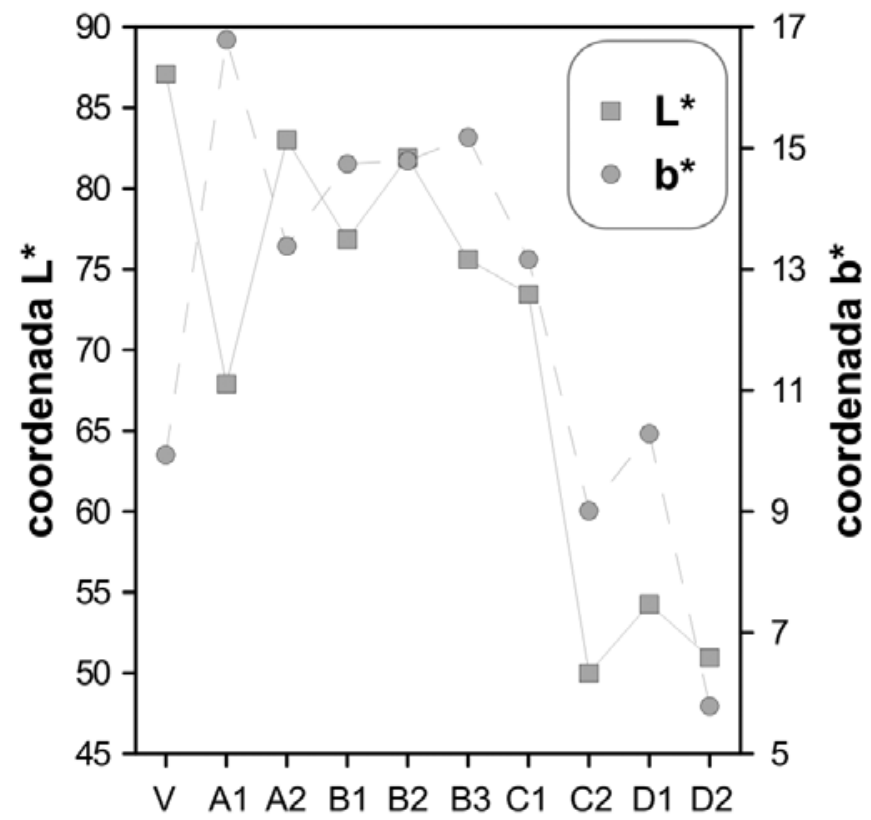

Figura 2: Coordenadas $L^{*} \mathrm{y} \mathrm{b}^{*}$ del vidriado transparente, y de los vidriados que incorporaban los pigmentos. 
se hubiese formado cierta proporción del complejo $\mathrm{Br}_{4} \mathrm{Au}$ que se caracteriza por un color rojizo (pero no podía ser mayoritario ya que no se había introducido suficiente $\mathrm{NH}_{4} \mathrm{Br}$ para ello). Tras la adición del coloide de sílice y el amoníaco, el color rojizo se mantuvo, sin que se apreciase la formación de heterogeneidades, lo cual indicaba que ambos reactivos eran compatibles con el producto coloreado ya formado. Tras el secado de las suspensiones se obtuvieron materiales de aspecto homogéneo, que tras la calcinación adquirieron un color rosado.

En los tres experimentos del segundo bloque (B1, B2 y B3), la presencia del $6 \mathrm{MH}$ alteró la evolución del sistema, tendiendo a generar una suspensión con diferentes tonos de marrón, y más bien opaca. De ello se infería que el $6 \mathrm{MH}$ podría favorecer la coagulación de las AuNPs antes de que pudiesen interaccionar con el coloide de sílice. La heterogeneidad de la suspensión se mantuvo en los materiales de color marrón rojizo obtenidos tras el secado, pero tras la calcinación se obtuvieron materiales de tono rosado relativamente homogéneo.

Durante la síntesis del pigmento $\mathrm{C} 1$ el comportamiento fue idéntico al descrito para el A1, y el aspecto de la suspensión no varió cuando finalmente se incorporó la DTA. Durante el secado de la suspensión $\mathrm{C} 1$ se apreció que no se generaban heterogeneidades en el sólido, y tras la calcinación se obtuvo un material de color rosado. En el caso del pigmento C2 el comportamiento durante la reacción fue idéntico, sin que se apreciasen cambios en el aspecto de la suspensión tras la adición de la disolución de $\mathrm{CMC}$, de lo que se dedujo que este aditivo tampoco afectaba a la estabilidad de la suspensión de sílice y AuNPs. Sin embargo, tras el secado se obtuvo un material de aspecto violáceo oscuro, completamente diferente a los obtenidos previamente.

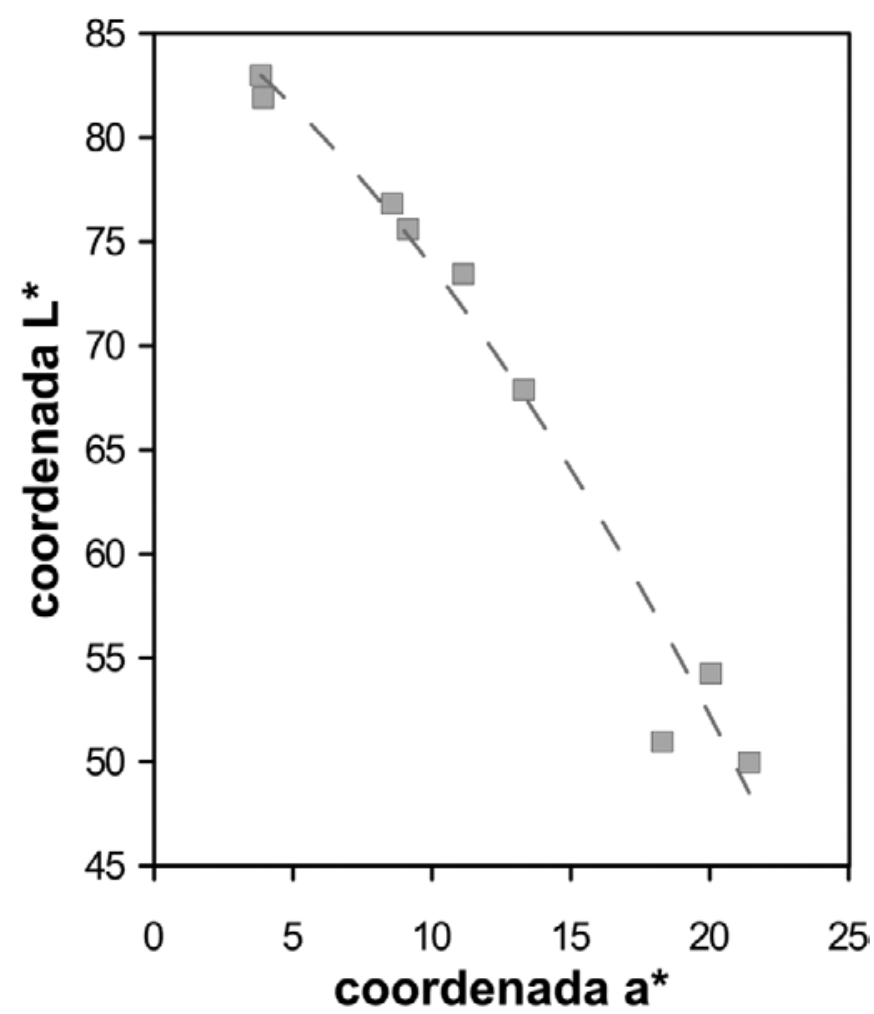

Figura 3: Relación de a* con $\mathrm{L}^{*}$ en los vidriados que incorporaban los pigmentos.
Durante la síntesis de los pigmentos D1 y D2 la secuencia de acontecimientos fue idéntica a la observada en el C2, de lo que se deduce que el cambio en la naturaleza del óxido protector no afectaba al desarrollo del proceso. En ambos casos, tras el secado se obtuvo un material de color violáceo oscuro.

\section{2 Desarrollo de color}

Todos los pigmentos sintetizados se incorporaron al vidriado transparente, obteniéndose una gama de tonos rojizos con saturaciones muy diferentes, tal como reflejan las coordenadas cromáticas de los mismos (Figura 1 y Figura 2). Asimismo, $\Psi$ también presentó un intervalo de variación muy amplio.

La evolución de las coordenadas cromáticas (y la de $\Psi$ que resultó prácticamente paralela a la de $a^{*}$ ), demuestra que el poder colorante del pigmento es muy sensible a las condiciones de síntesis. De los resultados obtenidos se pueden inferir los siguientes efectos de las variables estudiadas:

- Es posible sintetizar el pigmento sin incorporar aditivos orgánicos, pero el poder colorante es relativamente bajo, y sobre todo el aprovechamiento del oro es reducido. En estas condiciones, un incremento en la relación $\mathrm{SiO}_{2} /$ $\mathrm{Au}$ o en el tiempo de reacción, parecen ejercer un efecto perjudicial sobre el poder colorante del pigmento.

- La incorporación del $6 \mathrm{MH}$ en paralelo al $\mathrm{NH}_{4} \mathrm{Br}$ aparentemente mejora ligeramente el poder colorante del pigmento, tal como refleja los valores de $\Psi$ para los pigmentos B1 y B3, que son similares al conseguido con

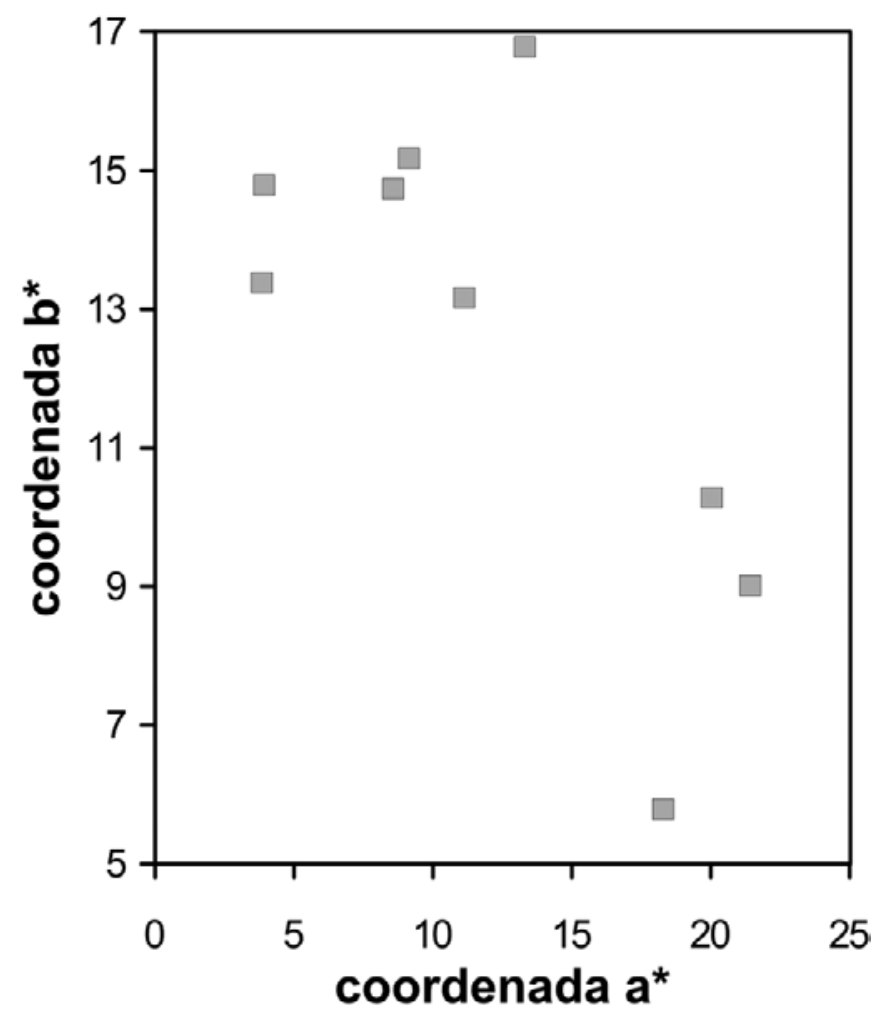

Figura 4: Relación de $\mathrm{a}^{*}$ con $\mathrm{b}^{*}$ en los vidriados que incorporaban los pigmentos. 
el A1 (aunque como se introdujo una menor cantidad de pigmento $\mathrm{a}^{*}$ resulte inferior). Por otra parte, parece adecuado mantener baja la concentración de $\mathrm{NH}_{4} \mathrm{Br}$ durante la reacción de síntesis de las AuNPs, para conseguir un pigmento con mayor poder colorante, mientras que concentraciones elevadas de $\mathrm{NH}_{4} \mathrm{Br}$ no parecen convenientes.

- La incorporación de DTA al final del proceso para facilitar la interacción entre el coloide de sílice y las AuNPs parece ejercer un efecto favorable, ya que se consigue un incremento notable de $\Psi$ con respecto al pigmento A1. Sin embargo, la contribución más destacada al poder colorante del pigmento es la adición de CMC y la eliminación de la etapa de calcinación, ya que de este modo casi se triplica el valor de $\Psi$ con respecto al A1, consiguiendo además unos valores muy elevados de $\mathrm{a}^{*}$.

- Una vez identificadas unas condiciones aceptables de procesado, la naturaleza del óxido que forma la cápsula protectora no es el factor determinante del poder colorante del pigmento, ya que la sustitución del $\mathrm{SiO}_{2}$ por $\mathrm{Al}_{2} \mathrm{O}_{3} \mathrm{O}$ $\mathrm{SnO}_{2}$ como material de encapsulado también genera pigmentos con un poder colorante elevado, aunque algo inferior al del pigmento $\mathrm{C} 2$.

La luminosidad de los colores generados está fuertemente correlacionada con $\mathrm{a}^{*}$, de modo que los pigmentos que generan los colores más rojizos, también generan los menos luminosos (Figura 3), aunque de la tendencia que definen los datos experimentales apuntan a que existe un límite en el valor de $a^{*}$ que es posible obtener en el vidriado.

La componente amarilla de los vidriados no presenta una correlación clara con $a^{*}$ (Figura 4). Cuando el poder

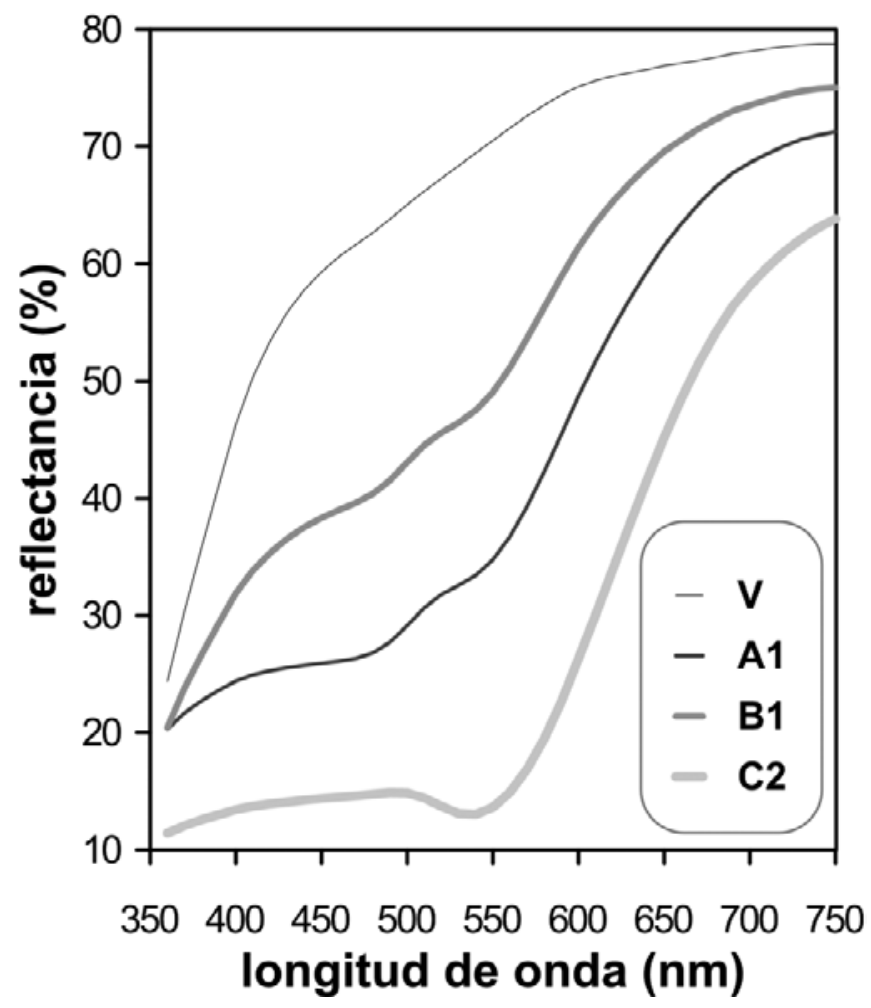

Figura 5: Curvas de reflectancia del vidriado transparente y de los vidriados que incorporaban algunos de los pigmentos sintetizados. colorante del pigmento es bajo o moderado, el $\mathrm{b}^{*}$ del color obtenido en el vidriado siempre es mayor que el del vidriado base. En cambio, cuando el poder colorante del pigmento es muy elevado, se llegan a conseguir valores de $b^{*}$ similares o inferiores a los del vidriado base, siendo el caso más destacado el pigmento D2, donde la combinación de las nanopartículas con la cápsula de $\mathrm{SnO}_{2}$ produce el valor más reducido de b*, y por tanto el rojo más puro.

Los espectros de reflectancia de los vidriados obtenidos (como ejemplos en la Figura 5 se comparan los del vidriado base con los correspondientes a los vidriados que contenían los pigmentos A1, B1 y C2, mientras que en la Figura 6 se comparan los vidriados que contenían los pigmentos C2, D1 y D2), indican que el color rojo se debe a una reducción en la reflectancia por debajo de $650 \mathrm{~nm}$, producida por una banda de absorción muy intensa centrada en $540 \mathrm{~nm}$ aproximadamente, pero que sólo aparece claramente definida en el caso del pigmento C2. Este resultado es coherente con la fuerte absorción característica del fenómeno de la RSP generado por las AuNPs (la longitud de onda de la máxima absorción de las AuNPs dispersas en agua varía entre 517 y $575 \mathrm{~nm}$ al aumentar su diámetro de 9 a $99 \mathrm{~nm}$ ) (13).

Los espectros justifican la falta de una correlación clara entre $b^{*} \mathrm{y} \mathrm{a}^{*}$, ya que en la zona del espectro correspondiente al amarillo, las curvas presentan la mayor pendiente, y por tanto pequeñas alteraciones afectan en mayor medida $a b^{*}$.

La banda de absorción de la RSP apenas varía al cambiar la sílice por alúmina (Figura 6), pero en el caso del $\mathrm{SnO}_{2}$ existe cierto desplazamiento hacia longitudes de onda superiores, que es la causa de la menor componente amarilla generada en el vidriado. Suponiendo que el tamaño de las nanopartículas es similar en los pigmentos C2, D1 y D2, esta variación se puede

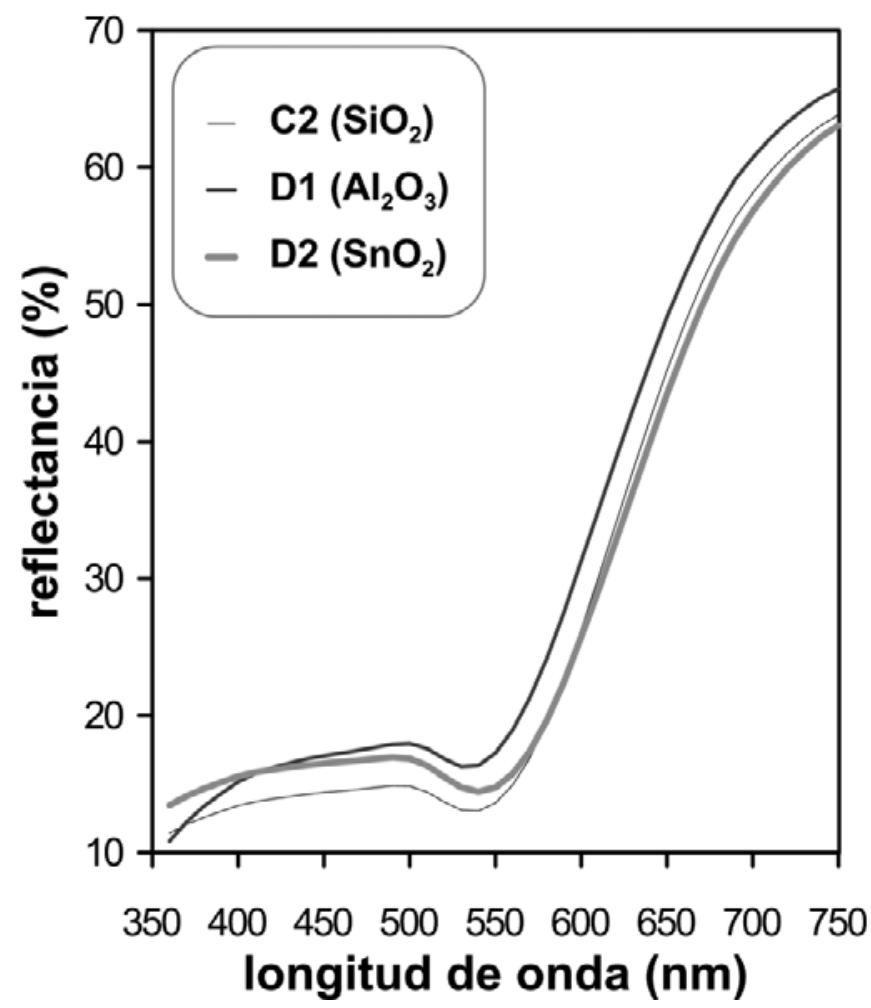

Figura 6: Curvas de reflectancia de los vidriados que incorporaban los pigmentos sintetizados con diferentes óxidos. 
interpretar considerando que la absorción de la RSP depende del índice de refracción del medio que rodea a las AuNPs, y la mayor diferencia se produce entre los índices de refracción del $\mathrm{SiO}_{2}$ y del $\mathrm{SnO}_{2}$ (1,54 frente a 2,00 respectivamente) (14).

\subsection{Microestructura de los pigmentos}

Las curvas espectofotométricas de los vidriados eran coherentes con la presencia de AuNPs en los pigmentos, pero se utilizó la microscopía electrónica de barrido para confirmar la presencia de las mismas. En el caso del pigmento B1 se identificaron partículas de oro aproximadamente esféricas dispersas en el seno de la sílice (Figura 7, izquierda), pero que presentaban una distribución de tamaños bastante ancha. La mayoría de las partículas áureas poseían un tamaño próximo a la micra y sólo una fracción muy pequeña poseía un tamaño inferior a $100 \mathrm{~nm}$ requerido para que la RSP tuviese lugar. Ello era coherente tanto con el color rosado del pigmento, como con su reducido poder colorante.

En el caso del pigmento C2, las AuNPs de tamaño inferior a $100 \mathrm{~nm}$ eran la fracción más numerosa (Figura 7 centro y derecha), y estaban muy bien dispersadas en el seno de la sílice, lo cual es acorde con su color violáceo y su elevado poder colorante.

Por otra parte cabe resaltar que la matriz silícea en el pigmento B1 presenta un grado de sinterización muy elevado debido al tratamiento térmico al que había sido sometido, mientras que en el C2 la matriz silícea estaba formada por nanopartículas bien diferenciadas, entre las cuales se distribuían las AuNPs. Es posible que el tratamiento térmico que permite sinterizar la sílice, también favorezca el crecimiento de las AuNPs (podría producirse un transporte de materia vía fase gas gracias a la mayor presión de vapor en equilibrio con la superficie de las partículas nanométricas), con lo cual la mayor parte de las partículas presentes en el pigmento final poseerían un diámetro superior al requerido.

\section{CONCLUSIONES}

- Es posible sintetizar pigmentos rojos para vidriados encapsulando AuNPs en una matriz de óxido refractario. Para conseguir un elevado poder colorante, es preciso que el diámetro de las AuNPs no supere los 100 nm, ni durante su síntesis, ni durante el proceso de encapsulado.

- Las AuNPs con las características deseadas se pueden sintetizar por reacción entre disoluciones acuosas de $\mathrm{HAuCl}_{4}$ y $\mathrm{NH}_{4} \mathrm{Br}$, operando de modo que la concentración de $\mathrm{NH}_{4} \mathrm{Br}$ se mantenga reducida durante la reacción.

- El encapsulado de las AuNPs se puede lograr adicionando el óxido protector en forma de dispersión coloidal a la suspensión de AuNPs (óxidos de Si, Al y $\mathrm{Sn}$ son adecuados para el proceso), y alcalinizando el medio para provocar la coagulación de las nanopartículas oxídicas. Se ha comprobado que la presencia de dietilentriamina durante la coagulación mejora el proceso de encapsulado

- El secado de la suspensión es un factor crítico para obtener el pigmento. Por ello conviene incrementar la viscosidad de la suspensión adicionando $\mathrm{CMC}$, para evitar la segregación y posterior aglomeración de las AuNPs. No resulta conveniente someter al pigmento a un tratamiento térmico a alta temperatura, ya que ello provoca el crecimiento de las AuNPs, y una merma de su poder colorante.

- Los pigmentos desarrollados poseen un poder colorante muy elevado en vidriados transparentes, generando diferentes tonos de rojo intenso, dependiendo del óxido que constituye la cápsula protectora.

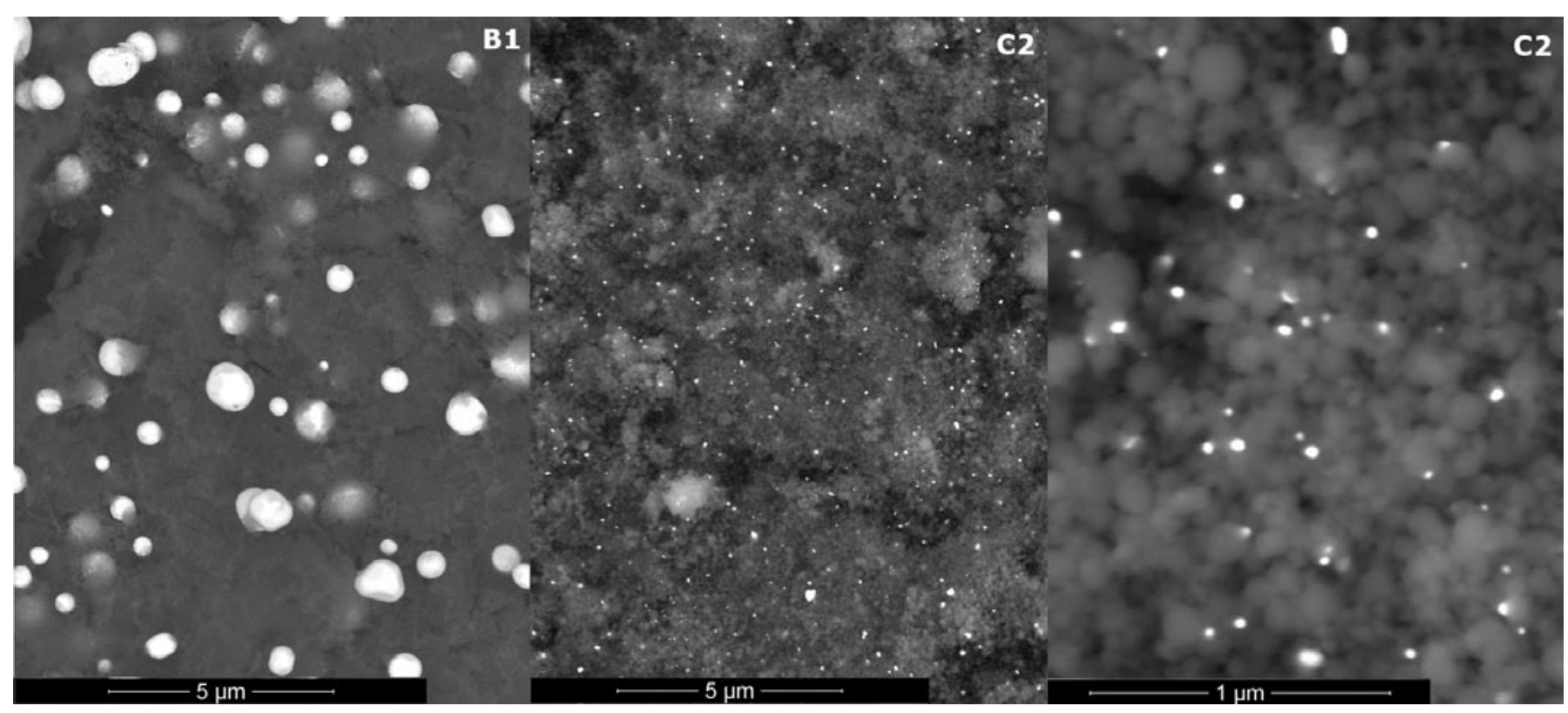

Figura 7: Fotografías de las partículas de los pigmentos B1 y C2 a los mismos aumentos (izquierda y centro), y del C2 a mayores aumentos (derecha). Las zonas blancas corresponden a las partículas de oro. 


\section{BIBLIOGRAFÍA}

(1) Singer F. , Singer S.S. . Industrial ceramics. London: Chapman \& Hall. 1963, p. 649.

(2) Cao G. Nanostructures $\mathcal{E}$ nanomaterials: synthesis, properties $\mathcal{E}$ applications. London: Imperial College Press. 2004, p..362.

(3) Sun Y. , Sia Y. Gold and silver nanoparticles: A class of chromophores with colors tunable in the range from 400 to $750 \mathrm{~nm}$. Analyst., 128, 686-691 (2003).

(4) Institutul de Cercetari Pentru Industria Sticlei si Ceramicii Fine. Procede pour obtenir des couleurs ceramiques en base d'or coloidale. RO64442A2, 1979-15-01.

(5) Herrrmann, H.J., Buettner, P. , W. List. Verfaren zur herstellung hochresistenter purpurfarben. DD143423A1, 1980-08-20.

(6) Cerdec AG. Purple pigments, their manufacture and their use. EP0501139B1, 1992-01-23.

(7) Dmc2 Degussa Metals Catalysts, Cerdec AG. Pigments for producing purple-coloured ceramic decorations, process for producing them and their use. EP0845506B1, 1997-11-12.

(8) Dmc2 Degussa Metals Catalysts, Cerdec AG. Spherical colour pigments, process for their preparation and their use. EP0845505B1, 1997-11-05.

(9) Sau T.K., Murphy C.J. Room temperature, high-yield synthesis of multiple shapes of gold nanoparticles in aqueous solution. J. Am. Chem. Soc. 126, 8648-8649 (2004).
(10) Vaskelis A., Tarozaité R., Jagminiené A., Tamasauskaité L., Juskenas R., Kurtinaitiené M. Gold nanoparticles obtained by $\mathrm{Au}(\mathrm{III})$ reduction with $\mathrm{Sn}(\mathrm{II})$ : Preparation and electrocatalytic properties in oxidation of reducing agents. Electrochimica Acta 53, 407-416 (2007).

(11) Brust M., Walker M., Bethell D., Schiffrin D.J., Whyman R. Synthesis of thiol-derivatised gold nanoparticles in a two-phase liquid-liquid system. J. Chem. Soc., Chem. Commun., 801-802 (1994).

(12) Zhang F. , Srinivasan M.P. Layer-by-layer assembled gold nanoparticle films on amine-terminated substrates. J. Colloid Interface Sci. 319, 450-456 (2008)

(13) Link S., M.A. El-Sayed. Size and temperature dependence of the plasmon absorption of colloidal gold nanoparticles J. Phys. Chem. B103, 4212-4217 (1999).

(14) Lide D.R. (Ed.) CRC handbook of chemistry and physics. $88^{\text {th }}$. Boca Raton: CRC, 2008.

Recibido: 01/03/2012

Aceptado: $11 / 04 / 2012$ 